\title{
Should You Sleep on It? The Effects of Overnight Sleep on Subjective Preference-based Choice
}

\section{Citation}

Karmarkar, Uma R., Baba Shiv, and Rebecca M.C. Spencer. "Should You Sleep on It? The Effects of Overnight Sleep on Subjective Preference-based Choice." Journal of Behavioral Decision Making (forthcoming). (Available online early.)

\section{Published Version}

http://onlinelibrary.wiley.com/doi/10.1002/bdm.1921/full

\section{Permanent link}

http://nrs.harvard.edu/urn-3:HUL.InstRepos:28548031

\section{Terms of Use}

This article was downloaded from Harvard University's DASH repository, and is made available under the terms and conditions applicable to Open Access Policy Articles, as set forth at http:// nrs.harvard.edu/urn-3:HUL.InstRepos:dash.current.terms-of-use\#OAP

\section{Share Your Story}

The Harvard community has made this article openly available.

Please share how this access benefits you. Submit a story.

\section{Accessibility}


Should you sleep on it? The effects of overnight sleep on subjective preferencebased choice

\author{
Uma R. Karmarkar ${ }^{1}$, Baba Shiv ${ }^{2}$, and Rebecca M. C. Spencer ${ }^{3}$ \\ ${ }^{1}$ Harvard Business School, Cambridge, MA, U.S.A. \\ ${ }^{2}$ Stanford Graduate School of Business, Stanford, CA, U.S.A. \\ ${ }^{3}$ Department of Psychology, University of Massachusetts, Amherst, MA, U.S.A
}


Conventional wisdom and studies of unconscious processing suggest that sleeping on a choice may improve decision-making. Though sleep has been shown to benefit several cognitive tasks, including problem solving, its impact on everyday choices remains unclear. Here we explore the effects of "sleeping on it" on preference-based decisions among multiple options. In two studies, individuals viewed several attributes describing a set of items and were asked to select their preferred item after a 12-hour interval that either contained sleep or was spent fully awake. After an overnight period including sleep, individuals showed increases in positive perceptions of the choice set. This finding contrasts with previous research showing that sleep selectively enhances recall for negative information. In addition, this increase in positive recall did not translate into a greater desire to purchase their preferred item or into an overall benefit for choice satisfaction. Time-of-day controls were used to confirm that the observed effects could not be explained by circadian influences. Thus we show that people may feel more positive about the choice options, but not more confident about the choice after "sleeping on" a subjective decision. We discuss how the valence of recalled choice set information may be important in understanding the effects of sleep on multi-attribute decision making, and suggest several avenues for future research. 
When struggling with a multi-faceted decision, conventional wisdom suggests "sleeping on it" will help. This belief has been advanced by studies demonstrating improved likelihood of making an optimal choice when people spend time away from the choice and information related to it (Dijksterhuis, 2004; Dijksterhuis, Bos, Nordgren, \& van Baaren, 2006; Lerouge, 2009). Such benefits have been attributed to unconscious processing of the relevant information, providing an increased capacity to examine tradeoffs between options, and better weighting and integration of attributes and emotional responses (Bos, Dijksterhuis, \& van Baaren, 2011; Dijksterhuis, 2004; Dijksterhuis et al., 2006; Strick et al., 2011 but see Newell, Wong, Cheung, \& Rakow, 2009; Nieuwenstein et al., 2015; Payne, Samper, Bettman, \& Luce, 2008b). However, most of these studies did not involve the experience of actually sleeping on a decision. In those experiments that did include multi-day intervals (e.g. Dijksterhuis et al. 2006), the comparison of interest was related to thinking styles rather than comparing sleep to wake. Here we consider the specific influence of sleep during the offline interval.

Recent studies suggest a meaningful positive role for sleep in at least one form of decision-making. For example, sleep deprivation impairs performance on the Iowa Gambling Task (IGT), a multi-round set of choices in which participants are given little initial information and thus must infer the optimal strategy through experience over time (Killgore, Balkin, \& Wesensten, 2006). Sleep deprived individuals made riskier choices, and obtained lower winnings overall, despite receiving an occasional high value payout. Such a result could reflect changes in attentiveness or motivation that accompany deprivation. However, this shift in strategy is also consistent with work demonstrating that sleep-deprived individuals shift towards seeking gains and away from defending against losses in risky choices, independent of changes in vigilant attention (Venkatraman, Huettel, Chuah, Payne, \& Chee, 2011). Contrasting with these detrimental effects of deprivation, participants specifically made more advantageous choices on the IGT following an interval containing overnight sleep relative to an equivalent interval of wake (Pace-Schott, Nave, Morgan, \& Spencer, 2011).

While studies directly examining the role of normal sleeping behavior on decision-making are quite limited, periods of sleep have been shown to benefit several processes intimately related to choice. Perhaps the most substantial contribution of this 
literature is a corpus of work supporting a role of sleep on memory. Recall on a range of memory tasks is greater following an interval with sleep (e.g., overnight sleep, mid-day nap) compared to recall following an equivalent interval awake (for reviews, see:

Diekelmann, Wilhelm, \& Born, 2009; Spencer, 2013). This benefit of sleep on memory is thought to reflect the reactivation of memories during sleep. For instance, recall of word pairs is greater following a 12-hr interval containing about 6 hours of overnight sleep compared to recall following a 12-hr daytime interval spent awake (Baran, Wilson, $\&$ Spencer, 2010). Such differences cannot be accounted for by differences in the timeof-day at which recall is tested, as immediate recall does not differ for groups who encode the word pairs in the morning and those that encode in the evening. Moreover, post-sleep performance is not associated with time spent asleep, as may be expected if sleep merely protected the memory from waking interference. Rather, post-sleep performance correlates specifically with time spent in slow wave sleep, a sleep stage which supports hippocampal-neocortical (i.e., short-term to long-term) transfer of memories via the co-occurrence of hippocampal ripples and neocortical spindles (Siapas \& Wilson, 1998). The resulting stabilization of memories, occurring preferentially over sleep, is termed sleep-dependent memory consolidation (Spencer, 2013).

Recent literature has also emphasized a broader role of sleep in cognitive processing. Experiencing distinct sleep phases has been shown to be conducive to “cognitive flexibility” as seen in successfully solving word puzzles (Walker, Liston, Hobson, \& Stickgold, 2002). More directly, sleep has been shown to increase creativity and the ability to devise creative solutions to problems (Cai, Mednick, Harrison, Kanady, \& Mednick, 2009). The opportunity to "sleep on" new information or tasks also benefits gist extraction (Payne, Stickgold, Swanberg, \& Kensinger, 2008a), and insight (the “aha!" moment) in solving problems with hidden rules (Wagner, Gais, Haider, Verleger, \& Born, 2004).

These effects predict that a period of time including sleep might also enhance subjective decision-making contexts, such as conventional shopping situations. In many of these, the optimality or quality of a decision involving multiple options is dependent on individual preferences. One source of difficulty in this type of choice lies in processing all of the relevant information, including making tradeoffs between attributes. 
Sleep could aid such decisions by increasing knowledgeability about the options in a manner similar to that described for unconscious processing (Dijksterhuis, 2004).

Previous work also suggests that sleep may preferentially strengthen emotionally salient, negative memories (Nishida, Pearsall, Buckner, \& Walker, 2009; Payne et al., 2008a; Wagner, 2001 but see Baran, Pace-Schott, Ericson, \& Spencer, 2012; Lewis, Cairney, Manning, \& Critchley, 2011). While these studies often examine the impact of more strongly affect laden images (e.g. a striking snake) rather than choice attributes, they do suggest that sleep could increase the relative weighting of negative over positive attributes. More broadly they raise the question of how sleep might interact with the valence of decision-relevant information, and/or affect valence-specific recall in ways that impact choice.

We often encounter choices with information about multiple options, and then return to it to make a firm decision after some period of time, whether it is later in the day or overnight. During this delay, perceptions of the information and even initial preferences may be malleable and differently influenced by sleep or wake. While there has been some investigation of how sleep may influence decisions with an objectively "correct" outcome, there is little work on everyday consumer choices based on subjective preferences. In addition, given the findings of the unconscious processing literature, such research could help distinguish between the benefits of spending time away from a decision and actually "sleeping on it." Thus, here we examine the influence of a normal overnight period's activities (including sleep) as compared to the influence of activities during the day for a preference-based choice with no objectively optimal option. In two studies, participants were given positive and negative attributes of multiple items and then asked to make an incentive compatible choice following a 12-hr interval containing sleep or an equivalent waking interval. We compared the effects of intervals with (Sleep group) and without (Wake group) sleep on recall for choice set attributes, product perceptions, and perceptions of the choice itself. 


\section{Ethics Statement}

Institutional Review Board (IRB) approval was obtained at Stanford University prior to all studies. All participants provided written consent following description of the procedures.

\section{EXPERIMENT 1}

\section{Materials and Methods}

Participants

Sixty-two individuals (ages 18-36 yrs) successfully completed both sessions of the experiment in exchange for monetary compensation. Participants in the Wake group $(n=34)$ performed the first session between 8-10 AM and returned following a 12-hr daytime interval spent awake for session 2. Participants in the Sleep group $(n=28)$ performed the first session between 8-10 PM and returned following a 12-hr interval containing overnight sleep. Participants did not receive any instructions regarding sleep. At the experiment's close, participants answered a short questionnaire about their recent sleep behavior including how long they had slept between the two experimental sessions. Participants in the Sleep group reported $6.16 \mathrm{hrs}(\mathrm{SD}=1.27)$ of overnight sleep on average. Seven individuals in the Wake group who noted they had napped between sessions were excluded from analysis, leaving a Wake sample size of $n=27$.

\section{Session 1 Procedure}

At the start of the experiment, participants were instructed that they would choose between four laptop satchels at the end of session 2. They were informed that they would be viewing true information about the satchels during the course of the first session, and that their eventual choice would have potential personal consequences. Specifically, a subset of participants selected by random drawing would receive their chosen satchel. To begin the task, participants viewed images of the satchels, labeled with brand names, for a self-determined length of time.

Subsequently, participants were presented 36 stimuli consisting of a brand name and photo of the satchel paired with a product attribute (see Supplementary Table 1). 
Attributes were selected for each brand from retail descriptions and online consumer feedback in line with the incentive compatible nature of the study. Each laptop satchel was described by six nominally positive attributes (e.g. "trendy" or "spacious") and three nominally negative ones (e.g. "not waterproof"). The asymmetric allocation of attribute valence arose from previous work finding that negative information can have a profound and disproportionate influence on perceptions of an item (e.g. Peeters \& Czapinski, 1990) and can be more influential on opinions and memory in general (Baumeister, Bratslavsky, Finkenauer, \& Vohs, 2001; Rozin \& Royzman, 2001). Thus more positive than negative attributes were included to keep the overall value of the choice set high and to maintain participants' engagement with the decision process.

Each brand/attribute pair was presented on the screen for 5 seconds in a randomized order. After viewing all pairs, participants were immediately asked to rate all four satchels independently on how much they liked the satchel (1 [not at all] to 11 [very much]), whether they would buy the satchel themselves (scale of 1 [definitely no] to 11 [definitely yes]), and how interested they would be in owning the satchel (scale of 1 [not interested at all] to 11 [extremely interested]). Since individuals can be more reluctant to revisit the decision process when they perceive a choice to be "closed" or completed (Gu, Botti, \& Faro, 2013), during this session, participants were not asked to indicate or commit to a choice between the items.

Following a short break (10-15 mins), during which they worked on unrelated questionnaires, participants completed a free recall task. They were asked to list as many of the brand/attribute pairings as they could remember. Participants then self-rated the valence of each recalled pair by assigning them one of the following three labels: positive, negative or neutral. Six participants (3 Wake, 3 Sleep) logged these valence ratings incorrectly by rating the brands overall as opposed to rating each brand/attribute pair independently. For these participants, attribute valences were determined by consensus from three independent coders blind to study purpose and participant group.

\section{Session 2 Procedure}

Upon returning 12-hrs later, participants reviewed the satchel photos and repeated the satchel ratings, free recall, and valence rating procedures. Participants then chose 
their preferred satchel and rated the ease of making the decision, how confident they felt about the decision, and how satisfied they felt about the decision on scales of 1 [not at all] to 11 [extremely]. Responses across these three scales were highly consistent $(\alpha=.92)$.

\section{Circadian Control}

An independent sample of forty-two participants (ages 18-26 yrs) took part in a single-session control experiment, signing up for sessions between 8-10 AM (Morning control group; $n=21$ ) or 8-10 PM (Evening control group; $n=21)$. These participants first completed the session 1 experimental procedures described above. Following this, participants selected their preferred satchel, rated the perceived quality of their decision and responded to the sleep questionnaire. One participant who reported 24 hours of sleep deprivation was excluded from the Evening group, leaving a sample size of $n=20$. Decision quality measures (ease, confidence, and satisfaction) remained consistent $(\alpha=.899)$.

\section{Results}

In everyday contexts, information can be interpreted and encoded via a number of individual biases as the decision process unfolds (Russo, Medvec, \& Meloy, 1996; Russo, Meloy, \& Medvec, 1998). Thus, participants received no feedback about the accuracy of their recalled attributes in the first or second session, to capture self-generated product perceptions. The Wake group listed an average of $16.33(S D=4.44)$ total attributes in the first session, and $14.37(S D=4.30)$ attributes in the second session. The Sleep group listed $14.07(S D=5.38)$ items on average in the first session and $13.82(S D=5.285)$ attributes in the second session. To measure the effects of overnight sleep, we calculated the intersession change (e.g. the difference between session 2 and session 1) in number of brand/attribute pairs listed for each participant. There was a significant main effect of sleep on the intersession changes in attributes listed, such that the Sleep group's recall decreased significantly less than the Wake group's $\operatorname{did}\left(F(1,53)=4.727, p<.05, \eta_{\mathrm{p}}{ }^{2}=.082\right.$, Figure 1A).

Given that participants did not receive feedback on their recall accuracy, it is useful to examine whether the effects of sleep were specifically due to correctly or 
incorrectly recalled brand/attribute pairs, where incorrect thoughts included information not part of the original list as well as attributes assigned to the wrong brand. We tested the correct vs. incorrect difference scores using a repeated-measures ANOVA with sleep condition as a between-subjects factor (Figure 1A). There was no interaction between accuracy and sleep $(F(1,53)=.001, p=.98)$, suggesting that sleep influenced rates of reporting correct and incorrect information similarly in this setting.

We used participants' own valence ratings to examine the amount of positive and negative information recalled as percentages of the total number of brand/attribute pairs recalled. Percentages were used to help control for the fact that total recall varied significantly between individuals - thus an increase of one positive attribute between sessions would be far more significant for an individual who only listed three attributes, compared to someone who listed twenty. Specifically, we used valence (percent positive and percent negative) and session number as repeated measures in an ANOVA with sleep as a between subjects factor to understand how sleep might change the balance of positive to negative perceptions. Table 1 provides a breakdown of how the recalled information was categorized, and the percentage of each type of information in both sessions. Examining the intersession change in these numbers, (e.g. the interaction between valence, session and sleep condition) the Sleep group showed a marginal trend in which the balance of positive to negative (self-rated) information shifted more towards positive information over sessions (Figure 1B, $F(1,53)=3.510, p=.067, \eta_{p}{ }^{2}=.062$ ).

If a participant attributed relatively more positive information to the items in the choice set following sleep, it is possible that there was also a positive change in their affective response or liking ratings for choice set as well. We first calculated the intersession difference in average liking for the choice set. A univariate ANOVA found no significant main effect of sleep condition on this difference score (Figure 2A; $F(1,53)=1.271, p=.265)$. However, repeating the analysis on liking for the preferred item, that is, the satchel that was eventually chosen by the participant, suggested a marginal increase in liking in the Sleep but not Wake group $\left(F(1,53)=3.841, p=.055, \eta_{p}{ }^{2}=.068\right)$.

In contrast, when similar difference scores were calculated for ratings of interest in buying the satchel, the Sleep group showed significantly less desire to purchase on average, compared to the Wake group (Figure $2 \mathrm{~B}, F(1,53)=6.588, p<.02, \eta_{p}{ }^{2}=.11$ ). 
Furthermore, though the Sleep participants reported higher liking for their preferred item, they indicated decreased intentions to purchase it if given the option to do so $\left(F(1,53)=9.383, p<.005, \eta_{p}{ }^{2}=.15\right)$. There was no effect of sleep, compared to wake, on intersession changes in "interest in owning" the satchels on average $(F(1,53)=.809, p=$ $.373)$ or for the preferred item $(F(1,53)=.654, p=.422)$.

Finally, participants explicitly selected which item they wanted to receive. Participants' selections ranged across all four satchel choices in both conditions, with each bag chosen by at least 10 out of the 55 participants, confirming that there was no globally dominating option in the set that might influence the ease of the choice. After making their choice, individuals in the Sleep condition reported significantly lower perceived decision quality compared to Wake as measured by the composite measure of ease, confidence and satisfaction $\left(F(1,53)=10.064, p<.004, \eta_{p}{ }^{2}=.16\right.$, Figure 2C). These results also show that remembering more information, or having more positive perceptions of the choice options following sleep compared to wake did not make the decision process more rewarding.

\section{Time of Day Controls}

While these findings suggest there may be some benefits for sleep on decisionmaking, this interpretation is vulnerable to concerns that participants may have confidence levels that vary in step with time of day, or generally more attention, motivation, or even self-control in the morning. Thus, similar to previous studies (PaceSchott et al., 2011) we assessed whether such circadian effects could account for the differences observed between Sleep and Wake groups by comparing the performance of Morning and Evening control groups. Most central to our findings, there were no differences in perceived decision quality overall related to session time $\left(M_{\text {Morning }}=7.80\right.$, $S D=2.16 ; M_{\text {Evening }}=7.95, S D=2.31$; repeated-measures ANOVA $\left.F(1,39)=.04 ; p=.842\right)$ nor were there differences for contrasts of any of the individual measures ( $p=.6$ and above). The average number of product attributes listed was not significantly different between the Morning $(M=13.29, S D=5.44)$ and Evening groups $(M=11.90, S D=3.52 ; F(1,39)$ $=.926, p=.34)$. In addition, there was no interaction between time of day and the relative 
percentages of positive and negative attributes listed $(F(1,39)=.60, p=.44$, repeatedmeasures ANOVA).

Ratings averaged across the choice set and for only preferred items are reported in Table 2. Liking and owning ratings did not differ between Morning and Evening groups. While desire to purchase did appear to be lower in the Morning compared to Evening group on average, importantly this difference was not significant for the preferred item. Thus, time-of-day cannot account for changes we observed in memory and decisionrelated perceptions following a period of normal sleep behavior.

Taken as a whole, the findings here suggest that "sleeping on" information relevant to a choice may have marginally positive influences on perceptions of the choice set and for the preferred option. This is distinctly different from the results of previous sleep studies, that find recall for negative information or images (e.g. Nishida et al., 2009; Payne et al., 2008a; Wagner, 2001). However, these increased positive thoughts did not translate into increasing commitment to the preferred option beyond the Wake group, as indicated by participants' levels of interest in buying their preferred satchel themselves. In addition, the Sleep group did not show any indication that they were more satisfied with their choice or that they felt they had made a better choice, counter to the predictions of the literature.

Given this disparity, it is worth considering whether the observed trends for valence were simply due to participants initially receiving more positive than negative information about the satchels. While this was done deliberately to increase engagement with the choice, and to better reflect the information offered in most everyday shopping choice situations, it could also have created an attentional bias towards positive attributes that was amplified by more general consolidation effects of sleep. Thus we conducted a second study to examine how these effects might be expressed in situations where equal amounts of positive and negative information were available, and to clarify the effects of sleep for recall in a decision-driven context more generally. 


\section{EXPERIMENT 2}

\section{Methods and Materials}

\section{Participants}

Forty individuals (ages 18-36 yrs) completed both sessions of the experiment in exchange for monetary compensation. Participants in the Wake group performed the first session between 8-10 AM and returned following a 12-hr daytime interval spent awake for session 2. Two Wake participants who indicated sleeping during the day were removed from the analysis, leaving a sample size of $n=19$ for this condition.

Participants in the Sleep group performed the first session between 8-10 PM and returned following a 12-hr interval containing overnight sleep. One participant from the Sleep group who slept for $<2$ hrs was excluded, leaving a sample size of $n=18$. The average self-reported sleep time between sessions for this group was $6.88 \mathrm{hrs}(S D=1.30)$.

\section{Session 1 Procedure}

Procedures were similar to Experiment 1; participants were instructed that they would choose between four laptop satchels at the end of session 2, and that their choice would be consequential (i.e., incentive compatible). Participants then viewed an image of the satchels, labeled with brand names, for a self-determined length of time.

Subsequently, they were presented stimuli that consisted of a brand name and photo of the satchel paired with a product attribute. Attribute valence was estimated from a pre-test in which an independent sample of 30 participants from the same general participant pool rated several attributes on a scale from 1 [strongly negative] to 9 [strongly positive]. For each laptop satchel we selected four positive (pre-rated mean $>6.7$ ) and four negative (pre-rated mean <3.9) attributes for a total of 32 brand/attribute pairs (see Supplementary Table 2). Attributes were assigned to brands such that they reflected accurate information about the satchel, maintaining incentive compatibility. Each attribute/brand pair was presented on the screen for 5 seconds in a randomized order.

After viewing all pairs, participants were immediately asked to rate all four satchels independently on how much they liked the satchel, whether they would buy the 
satchel themselves, and how interested they would be in owning the satchel. During this session, participants were not asked to indicate or commit to a choice between the items.

Following a short break (10-15 mins) in which they worked on unrelated questionnaires, participants completed a free recall task, listing as many of the pairs as they could remember. Participants then self-rated the valence of each as positive, negative, or neutral.

\section{Session 2 Procedure}

Upon returning 12-hrs later, participants reviewed the satchel photos and repeated the free recall and valence rating tasks as well as indicating how much they liked, wished to buy, and wished to own each satchel. After choosing the satchel they wished to receive if selected by random drawing, participants rated the ease of making the decision, how confident they felt about the decision, and how satisfied they felt about the decision on scales of 1 [not at all] to 11 [extremely]. Responses across these decision perception scales were highly consistent $(\alpha=.93)$ and were averaged to form a composite index of perceived decision quality.

\section{Results}

The Wake group listed an average of 12.84 total attributes $(S D=4.40)$ in the first session, and 11.95 attributes $(S D=4.88)$ in the second session. The Sleep group listed $13.17(S D=5.09)$ attributes on average in the first session and $13.22(S D=4.61)$ attributes in the second session. The effects of overnight sleep were measured via the intersession change (e.g. the difference between session 2 and session 1) in the total number of brand/attribute pairs listed. In this experiment, the inter-session change in recall was not significantly better for the Sleep compared to Wake condition $(F(1,35)=$ $.626, p=.434)$.

Next, we examined participants' valence ratings of the brand/attribute pairs. As in Experiment 1, we focused on the amount of information explicitly labeled as positive and negative as percentages of the total number of pairs recalled. Table 3 provides a breakdown of how the recalled information was categorized, and the percentage of each type of information in both sessions. There was a significant interaction between 
condition (Sleep v. Wake), session, and valence (positive v. negative) in the percentage of recalled items across sessions (three way interaction: $F(1,35)=4.66, p<.05, \eta_{p}{ }^{2}=.118$; repeated-measures ANOVA). Figure 3A summarizes this finding, showing the intersession percentage differences for each valence for the Sleep and Wake groups. As in Experiment 1, participants remembered relatively more positive as compared to negative information if they slept between the sessions than if they remained awake, even though they had been initially presented with equal amounts of positive and negative information.

Again, despite the sleep period leading to an overall bias towards positive thoughts about the choice set, participants in the Sleep group showed significantly less increase in desire to purchase their preferred satchel across sessions compared to those in the Wake group (Figure 3B; $F(1,35)=4.42, p<.05, \eta_{p}{ }^{2}=.112$ ). In this study there was no significant difference between groups in the intersession difference in desire to purchase across the choice set as a whole $(F(1,35)=.151, p=.70)$. Intersession changes in liking across the choice set $(F(1,35)=1.326, p=.257)$ and for the preferred item $(F(1,35)=.20$, $p=.658)$ did not differ significantly between conditions, nor did interest in owning the satchels on average $(F(1,35)=.1 .074, p=.307)$ or for the preferred item $(F(1,35)=.206, p$ $=.653)$.

Participants' satchel choices ranged across the entire choice set, with each option being selected by at least 6 people. Examining the composite post-choice ratings of decision quality in session 2 showed that here, they were not significantly different between the Sleep $(M=8.35, S D=2.36)$ and Wake groups $(M=8.61, S D=2.47 ; F(1,35)$ $=.110, p=.74)$. While sleep did not significantly decrease perceptions of choice quality, it also failed to increase them. In addition, pooling data from Experiment 1 and 2 shows that perceived decision quality decreased significantly overall for the Sleep groups ( $M=$ $7.68, S D=2.33)$ compared to the Wake ones $(M=8.75, S D=1.87 ; \mathrm{F}(1,89)=5.726$; $\mathrm{p}<.02, \eta_{p}{ }^{2}=.060$ ). "Experiment" (e.g. 1 or 2 ) was included as a covariate in this analysis and was not significant $(\mathrm{F}(1,89)=.923, \mathrm{p}=.34)$. These findings are seemingly in conflict with the observed increase in positive thoughts and run counter to lay theory predictions of feeling better about a decision after "sleeping on it". 


\section{GENERAL DISCUSSION}

These results show that in the context of subjective, preference-based choice, sleep can increase positive perceptions of the choice set. However, perceived decision quality significantly decreased (Experiment 1) or at best remained unchanged (Experiment 2) after an overnight interval with sleep. Thus, people do not feel they are making a better decision at this time, counter to expectations raised by the common suggestion to 'sleep on it'. Relatedly, participants in both experiments expressed less interest in independently buying their preferred item when they had slept in the time between learning about their options and making a selection. Indeed, across experiments, perceived decision quality was correlated with purchase intentions towards the preferred (chosen) option in Session 2 (pooled $n=92$; Pearson's $r=.276, p<.01$, partialling out the effect of experiment.) These findings suggest that after sleep, participants were less committed to their preferred option, and/or less willing to envision spending their own money to receive it.

One explanation for these results might be that Sleep/Wake differences arose from circadian influences on cognition, mood, or confidence, since session 2 occurred in the morning for the Sleep group, but in the evening for the Wake group. The Morning and Evening control groups for the first experiment largely rule this out, finding no difference in decision quality or product attribute measures. While one set of ratings desire to purchase - did show a possible influence of session time across the choice set, this circadian effect was not expressed for participants' preferred items, despite there being a significant difference between Sleep and Wake groups which replicated across two studies. Thus with respect to the present study, time of day remains unable to account for our findings.

The potential for enhanced recall following intervals of sleep compared to wake is consistent with a corpus of literature supporting a benefit of sleep on memory (for reviews, see: Diekelmann et al., 2009; Spencer, 2013). To our knowledge though, the present study is one of the first to contrast negative and positive stimuli directly and suggests that in a decision context, positive stimuli may be preferentially retained over intervals with sleep. Studies of emotional memories have largely focused on negative 
compared to neutral stimuli, and find that sleep's beneficial effects on consolidation are greater (Payne et al. 2008a; Nishida et al. 2009; van der Helm et al., 2011) or at least the same (e.g. Baran et al., 2012; Cunningham et al., 2014) for negative memories (for review, see: Deliens, Gilson, \& Peigneux, 2014). Less is known of the consolidation of positive emotional stimuli, though one recent study suggests they may also be consolidated over sleep to a greater extent than matched, neutral stimuli (Chambers \& Payne, 2014).

Whether the preferential consolidation of positive over negative stimuli is specific to the choice-oriented content in the present study is an important area for further research. Previous studies of sleep's role on emotional memory have largely used pictures where the negative images suggest an immediate or salient threat, like a striking snake (e.g. IAPS stimuli). These are generally compared to neutral (e.g. a lamp) or positive items (e.g. a baby seal) that have little direct salience to the observer (van der Helm et al., 2011). In the present study, all of the attributes are personally relevant insofar as the choice is personally relevant. In addition, the affect arising from negative attributes in our study is far less salient than what might be evoked by a threatening image. Thus our results provide a useful contrast to previous work on sleep and memory, by examining information relevant to a specific decision within an everyday context as opposed to more extreme images with no real world consequences for the viewers.

The relationship between sleep and positive information could also explain some of the differences in the effects of sleep between Experiments 1 and 2. In Experiment 2, the absolute availability of positive information was reduced (from 6 attributes per satchel to 4 attributes per satchel), and the addition of another negative attribute meant that positive information made up a lesser percentage of the total information available (from $67 \%$ to 50\%). Such shifts may have reduced the engagement in the decision, or attractiveness of the items overall, as average choice set liking ratings were marginally lower in the first session of Experiment 2 compared to Experiment $1(\mathrm{p}=.054)$. The greater amount of negative information available in Experiment 2 also could have impacted the likelihood of postchoice regret arising from the unselected items.

The increase in positive (relative to negative) information recalled after sleep offers additional possible directions for future research. For example, though people 
response positively to having multiple attractive options, these types of "win-win" decisions between consumer products can also be accompanied by anxiety which increases as the value of the options increase (Shenhav and Buckner, 2014). Thus a positive bias in recall could increase the potential for choice anxiety or anticipated regret over un-chosen items, decreasing interest in purchase in a manner consistent with our findings.

Processing decision information during offline intervals has also been examined in several recent studies on unconscious, but waking, deliberation (Bos et al., 2011; Dijksterhuis, 2004; Dijksterhuis et al., 2006; Dijksterhuis \& van Olden, 2006; Lerouge, 2009; Strick, Dijksterhuis, \& van Baaren, 2010). In fact, if we consider the changes that occur in between sessions in the Wake group, the 12 hours spent apart from the choice information appears to increase purchase intentions towards the preferred item (Figures 2B and 3B), consistent with the benefits to choice demonstrated in that literature. However, as the effects of sleep in the present study are found in comparison to this Wake group, they likely extend beyond the potential contribution of unconscious processing.

Our measures and our decision setting, in which participants' inter-session behavior is not influenced or constrained, were designed to investigate the routine activity that might normally accompany a choice taking place over a longer time span. Thus it could be possible that reduced memory following daytime wake, as observed in Experiment 1, could be due to interference from other memories encoded over this interval rather than reflecting a sleep-dependent process such as memory consolidation. While we cannot rule out such an alternative, this explanation seems unlikely. In Experiment 1, the reported amount of time asleep for the sleep group is not significantly correlated with the intersession increase in recall (Pearson's $r=.277, p=.161$ ). In Experiment 2, increased time asleep is actually correlated with decreases in recall (Pearson's $r=-.479, p<.05$ ). In addition, previous studies in which participants encoded similar material such as word pairs (J. K. Wilson, Baran, Pace-Schott, Ivry, \& Spencer, 2012) and item-location pairs (Diekelmann, Büchel, Born, \& Rasch, 2011) have demonstrated that protection or enhancement of the memory over an interval with sleep is specifically associated with slow wave sleep. This suggests that it is time spent in this 
sleep stage, not merely time asleep and/or away from outside stimuli, that is critical to the sleep benefit. To better address this concern, however, it would be useful for future research to include both self-reports of activity during the intersession interval, as well as polysomnography ${ }^{1}$ in order to identify specific relevant stages of sleep.

Another alternate explanation for our results is that the participants in the Wake group had the opportunity to actively seek more information to help them make a choice about the laptop bags, or that they might have passively absorbed decision-relevant information by observing the bags of others. This could affect their perceptions of the decision, making them feel more knowledgeable and potentially more confident. It should be noted that both of these courses of action were possible for the Sleep group in both experiments as they only reported sleeping 6-7 hrs out of the roughly $12 \mathrm{hrs}$ intersession interval. In addition, for the Sleep groups, there was no correlation between duration of sleep time and average perceptions of decision quality (Experiment 1: Pearson's $r=-.094, p=.642$; Experiment 2: Pearson's $r=-.01, p=.98)$. However, their opportunities for gathering additional information were clearly more limited. While this distinction is one that arises naturally from a situation where a person chooses to sleep on a decision, it remains a limitation on assigning causality in the current study.

Finally, to choose the "best" option in the present study, individuals must determine which satchel fits their own preferences most closely. A period of sleep reduces the degree to which people believe they have made this match correctly, and the degree to which they believe they would purchase the item in the real world, but this study does not identify whether this predicts less happiness with their choice during ownership or consumption. Sleep broadly increased positive thoughts and specifically increasing liking for the chosen item. Such task-dependent enhancement of information would suggest that sleep could indeed lead to better decision quality overall when selecting one of several options, regardless of immediate subjective confidence. This may be particularly true for circumstances that require considering extensive amounts of information.

\footnotetext{
${ }^{1}$ A set of biophysical recordings that tracks signals such as brain EEG and eye movements, and is used to characterize sleep, sleep stages, and quality of sleep.
} 
In general, the impact of sleep deprivation on decision-making has received significant attention in various domains (e.g. Killgore et al., 2006; Killgore et al. 2007; Venkatraman et al., 2011). Understanding these effects is critical for understanding how sleep deprived individuals might make compromised choices, or ones that could lead to suboptimal outcomes. However, the focus on sleep deprivation risks missing out on the potential effects of normal sleep. In service of this goal, we believe that this study represents and important first step in understanding what ramifications such actions can have on elements in the decision process.

\section{CONCLUSION}

We used a task that captures elements of everyday choices based on individuals' own preferences to examine the influence of "sleeping on" a decision. Our results demonstrate that a time period that includes normal nighttime sleep has distinct and complex effects on several elements of the decision process. Sleeping on a decision engendered relatively more positive thoughts about the choice set. It might be assumed that this would make people feel better about their choice, and more interested in pursuing it. However, counter to predictions based on previous literature, as well as common assumptions, sleep failed to improve perceptions of decision quality and indeed seemed to make participants more reluctant to consider commitment to the preferred item (e.g. spending money to purchase it.) Given the novelty of these findings, it will be important for future research to investigate the effects of sleep on a broad range of decision types.

\section{ACKNOWLEDGEMENTS}

The authors would like to thank Rebecca Owen and Clare Bruzek for their assistance in data collection, and Marina Burke for her contribution to classifying the qualitative data. 


\section{REFERENCES}

Baran, B., Daniels, D., \& Spencer, R. M. (2013). Sleep-Dependent Consolidation of Value-Based Learning. PloS One, 8(10), e75326.

Baran, B., Pace-Schott, E. F., Ericson, C., \& Spencer, R. M. C. (2012). Processing of Emotional Reactivity and Emotional Memory over Sleep. Journal of Neuroscience, 32(3), 1035-1042. doi:10.1523/JNEUROSCI.2532-11.2012

Baran, B., Wilson, J., \& Spencer, R. M. C. (2010). REM-dependent repair of competitive memory suppression. Experimental Brain Research, 203(2), 471-477. doi:10.1007/s00221-010-2242-2

Baumeister, R. F., Bratslavsky, E., Finkenauer, C., \& Vohs, K. D. (2001). Bad is stronger than good. Review of General Psychology, 5(4), 323-370. doi:10.1037//10892680.5.4.323

Bos, M. W., Dijksterhuis, A., \& van Baaren, R. B. (2011). The benefits of "sleeping on things": Unconscious thought leads to automatic weighting. Journal of Consumer Psychology, 21(1), 4-8. doi:10.1016/j.jcps.2010.09.002

Cai, D. J., Mednick, S. A., Harrison, E. M., Kanady, J. C., \& Mednick, S. C. (2009). REM, not incubation, improves creativity by priming associative networks. Proceedings of the National Academy of Sciences, 106(25), 10130.

Chambers, A. M., \& Payne, J. D. (2014). Laugh yourself to sleep: memory consolidation for humorous information. Experimental Brain Research, 232(5), 1415-1427. doi:10.1007/s00221-013-3779-7

Cunningham, T. J., Crowell, C. R., Alger, S. E., Kensinger, E. A., Villano, M. A., Mattingly, S. M., \& Payne, J. D. (2014). Psychophysiological arousal at encoding leads to reduced reactivity but enhanced emotional memory following sleep. Neurobiology of Learning and Memory, 114(C), 155-164. doi:10.1016/j.nlm.2014.06.002

Deliens, G., Gilson, M., \& Peigneux, P. (2014). Sleep and the processing of emotions. Experimental Brain Research, 232(5), 1403-1414. doi:10.1007/s00221-014-3832-1

Diekelmann, S., Büchel, C., Born, J., \& Rasch, B. (2011). Labile or stable: opposing consequences for memory when reactivated during waking and sleep. Nature 
Neuroscience, 14(3), 381-386. doi:10.1038/nn.2744

Diekelmann, S., Wilhelm, I., \& Born, J. (2009). The whats and whens of sleep-dependent memory consolidation. Sleep Medicine Reviews, 13(5), 309-321. doi:10.1016/j.smrv.2008.08.002

Dijksterhuis, A. (2004). Think Different: The Merits of Unconscious Thought in Preference Development and Decision Making. Journal of Personality and Social Psychology, 87(5), 586-598. doi:10.1037/0022-3514.87.5.586

Dijksterhuis, A., \& van Olden, Z. (2006). On the benefits of thinking unconsciously: Unconscious thought can increase post-choice satisfaction. Journal of Experimental Social Psychology, 42(5), 627-631. doi:10.1016/j.jesp.2005.10.008

Dijksterhuis, A., Bos, M. W., Nordgren, L. F., \& van Baaren, R. B. (2006). On Making the Right Choice: The Deliberation-Without-Attention Effect. Science, 311(5763), 1005-1007. doi:10.1126/science.1121629

Gu, Y., Botti, S., \& Faro, D. (2013). Turning the Page: The Impact of Choice Closure on Satisfaction. Journal of Consumer Research, 40(2), 268-283. doi:10.1086/670252

Killgore, W. D., Balkin, T. J., \& Wesensten, N. J. (2006). Impaired decision making following $49 \mathrm{~h}$ of sleep deprivation. Journal of Sleep Research, 15(1), 7-13.

Killgore, W. D., Killgore, D. B., Day, L. M., Li, C., Kamimori, G. H., \& Balkin, T. J. (2007). The effects of 53 hours of sleep deprivation on moral judgment. Sleep-New York Then Westchester-, 30(3), 345.

Lerouge, D. (2009). Evaluating the Benefits of Distraction on Product Evaluations: The Mind-Set Effect. Journal of Consumer Research, 36(3), 367-379. doi:10.1086/599047

Lewis, P. A., Cairney, S., Manning, L., \& Critchley, H. D. (2011). The impact of overnight consolidation upon memory for emotional and neutral encoding contexts. Neuropsychologia, 49(9), 2619-2629. doi:10.1016/j.neuropsychologia.2011.05.009

Newell, B. R., Wong, K. Y., Cheung, J. C. H., \& Rakow, T. (2009). Think, blink or sleep on it? The impact of modes of thought on complex decision making. The Quarterly Journal of Experimental Psychology, 62(4), 707-732. doi:10.1080/17470210802215202

Nieuwenstein, M. R., Wierenga, T., Morey, R. D., Wicherts, J. M., Blom, T. N., 
Wagenmakers, E. J., \& van Rijn, H. (2015). On making the right choice: a metaanalysis and large-scale replication attempt of the unconscious thought advantage. Judgm. Decis. Mak, 10, 1-17.

Nishida, M., Pearsall, J., Buckner, R. L., \& Walker, M. P. (2009). REM Sleep, Prefrontal Theta, and the Consolidation of Human Emotional Memory. Cerebral Cortex, 19(5), 1158-1166. doi:10.1093/cercor/bhn155

Pace-Schott, E. F., Nave, G., Morgan, A., \& Spencer, R. M. C. (2011). Sleep-dependent modulation of affectively guided decision-making. Journal of Sleep Research, 21(1), 30-39. doi:10.1111/j.1365-2869.2011.00921.x

Payne, J. D., Stickgold, R., Swanberg, K., \& Kensinger, E. A. (2008a). Sleep Preferentially Enhances Memory for Emotional Components of Scenes. Psychological Science, 19(8), 781-788. doi:10.1111/j.1467-9280.2008.02157.x

Payne, J. W., Samper, A., Bettman, J. R., \& Luce, M. F. (2008b). Boundary conditions on unconscious thought in complex decision making. Psychological Science, 19(11), 1118.

Peeters, G., \& Czapinski, J. (1990). Positive-negative asymmetry in evaluations: The distinction between affective and informational negativity effects. European Review of Social Psychology, 1(1), 33-60.

Rozin, P., \& Royzman, E. B. (2001). Negativity bias, negativity dominance, and contagion. Personality and Social Psychology Review, 5(4), 296-320.

Russo, J. E., Medvec, V. H., \& Meloy, M. G. (1996). The distortion of information during decisions. Organizational Behavior and Human Decision Processes, 66(1), $102-110$.

Russo, J. E., Meloy, M. G., \& Medvec, V. H. (1998). Predecisional distortion of product information. Journal of Marketing Research, 438-452.

Shenhav, A., \& Buckner, R. L. (2014). Neural correlates of dueling affective reactions to win-win choices. Proceedings of the National Academy of Sciences, 111(30), 1097810983.

Siapas, A. G., \& Wilson, M. A. (1998). Coordinated interactions between hippocampal ripples and cortical spindles during slow-wave sleep. Neuron, 21(5), 1123-1128.

Spencer, R. M. C. (2013). Neurophysiological Basis of Sleep's Function on Memory and 
Cognition. ISRN Physiology, 2013(4), 1-17. doi:10.2466/PR0.100.2.613-626

Strick, M., Dijksterhuis, A., \& van Baaren, R. B. (2010). Unconscious-Thought Effects Take Place Off-Line, Not On-Line. Psychological Science, 21(4), 484-488. doi:10.1177/0956797610363555

Strick, M., Dijksterhuis, A., Bos, M. W., Sjoerdsma, A., van Baaren, R. B., \& Nordgren, L. F. (2011). A meta-analysis on unconscious thought effects. Social Cognition, 29(6), 738-762.

van der Helm, E., Yao, J., Dutt, S., Rao, V., Saletin, J. M., \& Walker, M. P. (2011). REM Sleep Depotentiates Amygdala Activity to Previous Emotional Experiences. Current Biology, 21(23), 2029-2032. doi:10.1016/j.cub.2011.10.052

Venkatraman, V., Huettel, S. A., Chuah, L. Y. M., Payne, J. W., \& Chee, M. W. L. (2011). Sleep Deprivation Biases the Neural Mechanisms Underlying Economic Preferences. Journal of Neuroscience, 31(10), 3712-3718. doi:10.1523/JNEUROSCI.4407-10.2011

Wagner, U. (2001). Emotional Memory Formation Is Enhanced across Sleep Intervals with High Amounts of Rapid Eye Movement Sleep. Learning \& Memory, 8(2), 112 119. doi:10.1101/lm.36801

Wagner, U., Gais, S., Haider, H., Verleger, R., \& Born, J. (2004). Sleep inspires insight. Nature, 427(6972), 352-355.

Walker, M. P., Liston, C., Hobson, J. A., \& Stickgold, R. (2002). Cognitive flexibility across the sleep-wake cycle: REM-sleep enhancement of anagram problem solving. Cognitive Brain Research, 14(3), 317-324.

Wilson, J. K., Baran, B., Pace-Schott, E. F., Ivry, R. B., \& Spencer, R. M. C. (2012). Sleep modulates word-pair learning but not motor sequence learning in healthy older adults. Neurobiology of Aging, 33(5), 991-1000.

doi:10.1016/j.neurobiolaging.2011.06.029 
Table 1: Average amounts and percentages of information recalled across valences in Experiment 1 (Standard deviations in parentheses).

\begin{tabular}{|l|c|c|c|c|}
\hline & \multicolumn{2}{|c|}{ Wake Group } & \multicolumn{2}{c|}{ Sleep Group } \\
\hline & Session 1 & Session 2 & Session 1 & Session 2 \\
\hline \# Positive & $7.63(3.44)$ & $7.00(3.11)$ & $7.25(3.77)$ & $7.68(3.74)$ \\
\hline \# Negative & $4.93(2.48)$ & $4.22(1.63)$ & $4.54(1.73)$ & $3.79(2.06)$ \\
\hline \# Neutral & $3.78(3.35)$ & $3.15(2.74)$ & $2.29(1.97)$ & $2.36(2.04)$ \\
\hline & & & & \\
\hline \% Positive & $46.6(16.1)$ & $48.1(15.0)$ & $50.0(13.5)$ & $56.5(16.1)$ \\
\hline \% Negative & $29.2(10.4)$ & $29.6(7.3)$ & $34.2(13.2)$ & $27.2(13.1)$ \\
\hline
\end{tabular}


Table 2: Mean Choice Set Ratings for Circadian Controls (standard deviation in parentheses)

\begin{tabular}{|l|c|c|c|c|c|c|}
\hline & \multicolumn{3}{|c|}{ Average For All Items } & \multicolumn{3}{c|}{ Preferred (Chosen) Item } \\
\hline & Morning & Evening & $\mathrm{p}$ & Morning & Evening & $\mathrm{p}$ \\
\hline Liking & $6.27(1.30)$ & $6.63(1.20)$ & .38 & $8.67(1.68)$ & $8.70(1.66)$ & .95 \\
\hline Would Buy & $3.85(1.82)$ & $5.06(1.93)$ & $<.05$ & $5.86(3.04)$ & $7.20(2.65)$ & .14 \\
\hline $\begin{array}{l}\text { Interest in } \\
\text { Owning }\end{array}$ & $5.54(1.79)$ & $6.04(1.47)$ & .34 & $8.00(2.21)$ & $8.35(2.41)$ & .63 \\
\hline
\end{tabular}


Table 3: Average amounts and percentages of information recalled across valences in Experiment 2 (Standard deviations in parentheses).

\begin{tabular}{|l|c|c|c|c|}
\hline & \multicolumn{2}{|c|}{ Wake Group } & \multicolumn{2}{c|}{ Sleep Group } \\
\hline & Session 1 & Session 2 & Session 1 & Session 2 \\
\hline \# Positive & $5.58(2.14)$ & $5.21(2.64)$ & $5.94(3.11)$ & $6.61(2.725)$ \\
\hline \# Negative & $3.95(2.35)$ & $3.63(2.43)$ & $5.17(2.31)$ & $4.72(2.63)$ \\
\hline \# Neutral & $3.47(2.25)$ & $3.16(2.41)$ & $2.06(1.69)$ & $1.89(1.61)$ \\
\hline & & & & \\
\hline \% Positive & $45.6(14.4)$ & $44.1(14.6)$ & $44.0(14.1)$ & $50.7(12.8)$ \\
\hline$\%$ Negative & $28.2(11.8)$ & $30.0(18.2)$ & $40.0(13.0)$ & $35.0(16.1)$ \\
\hline
\end{tabular}




\section{Figure Legends}

Figure 1: Experiment 1, Effects on Choice Set Information A) Overall intersession changes (session 2 - session 1) in recalled lists, and broken down by accurate and inaccurate brand/attribute pairs. B) Intersession changes in relative percentages of positive and negative information recalled. ${ }^{*} \mathrm{p}<.05,{ }^{\dagger} \mathrm{p}<.07$.

Figure 2: Choice Set Ratings and Decision Quality A) Intersession (session 2 - session 1) changes in liking ratings for all four products (Average) and only the product selected in the final decision (Preferred). B) Intersession changes in "interested in buying" ratings for all four products and only the product selected in the final decision. C. Differences in perceived decision quality (e.g. composite ratings of ease of making the decision, confidence and satisfaction with the choice). ${ }^{\dagger} p=.055,{ }^{*} p<.05, * * p<.005$

Figure 3: Experiment 2, Effects on Choice Set Information A) Intersession changes in relative percentages of positive and negative information recalled. B) Intersession changes in "interested in buy" ratings for all four products (Average) and only the product selected in the final decision (Preferred) $* \mathrm{p}<.05$. 
Figure 1

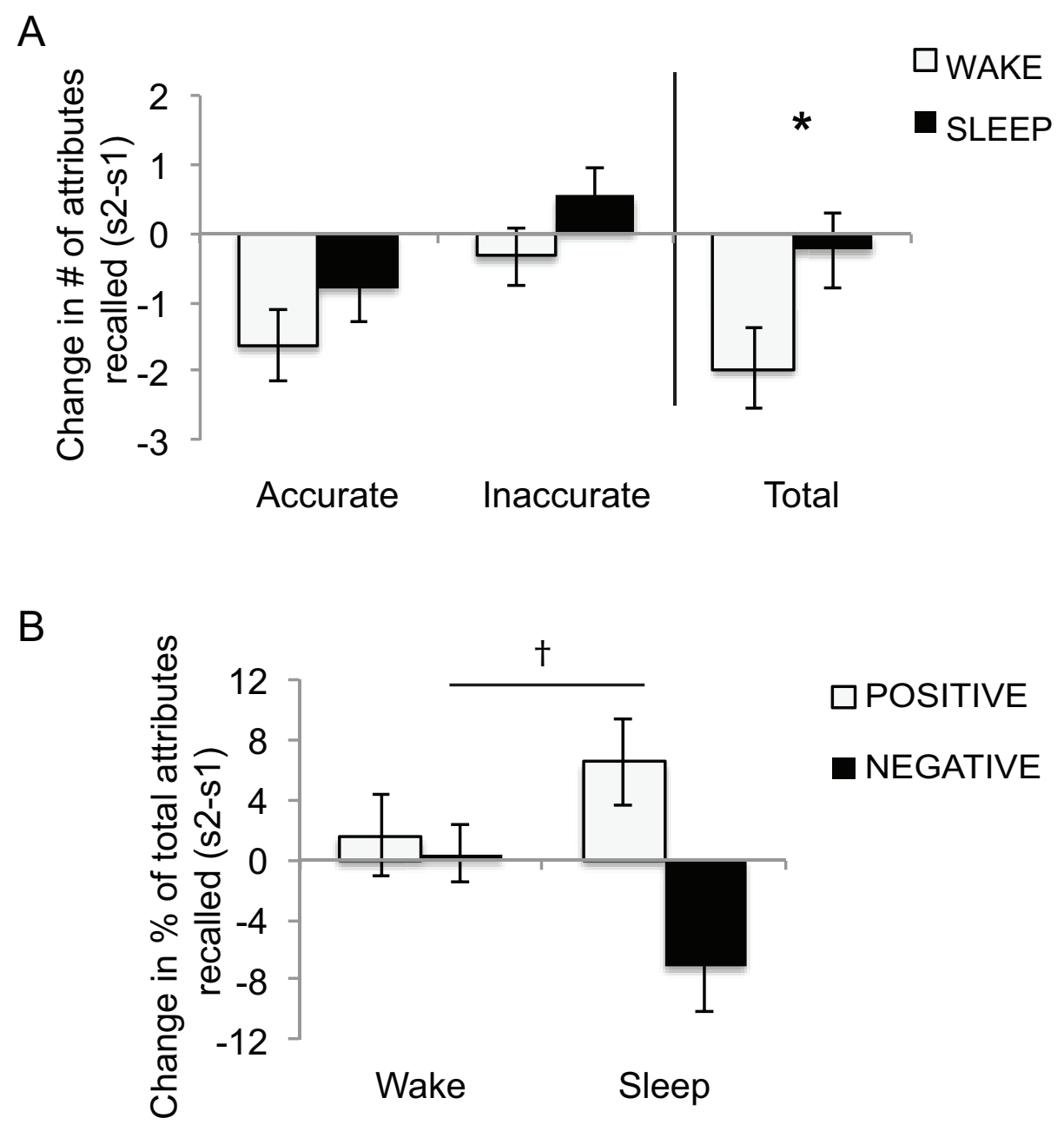


Figure 2
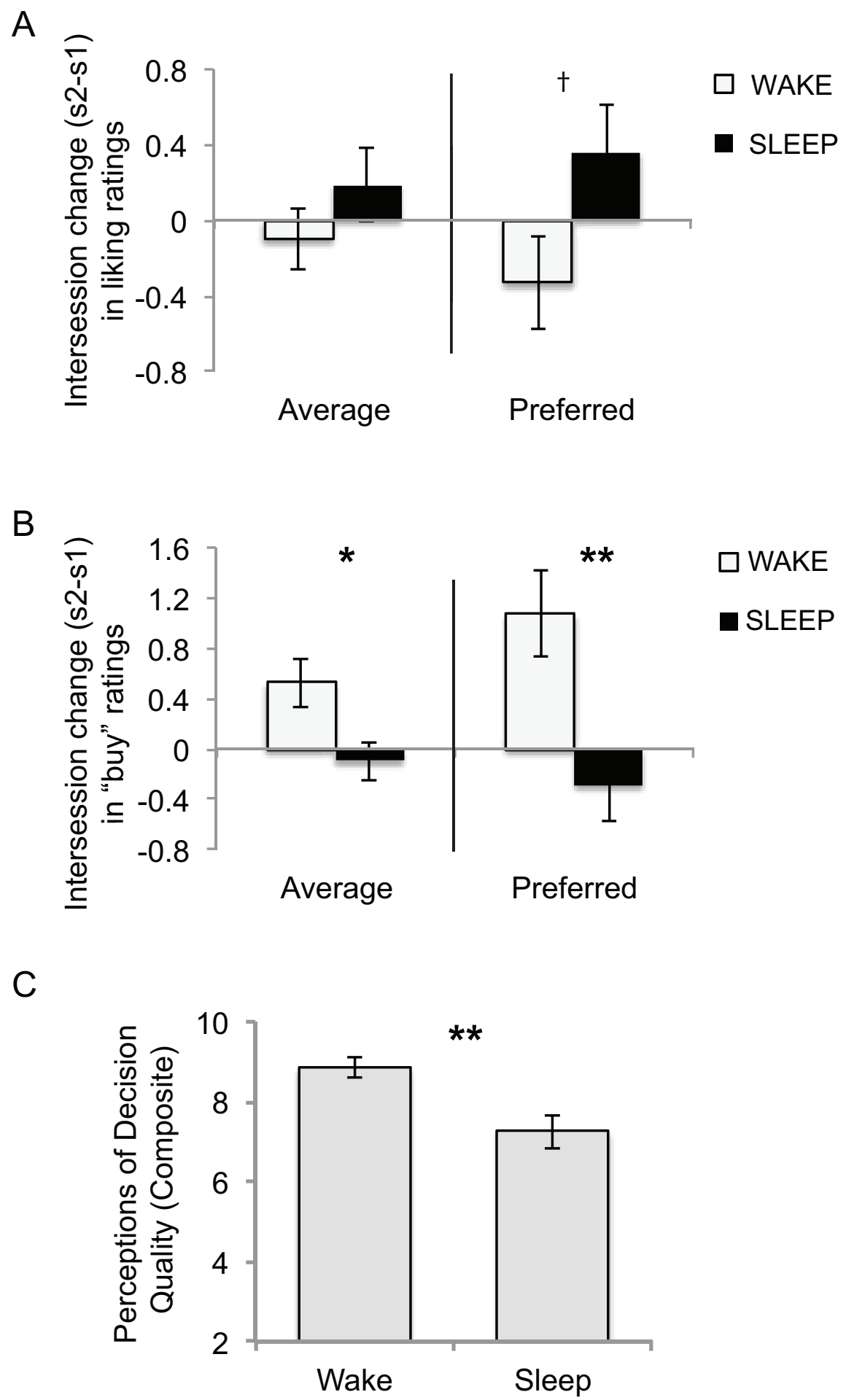
Figure 3

A

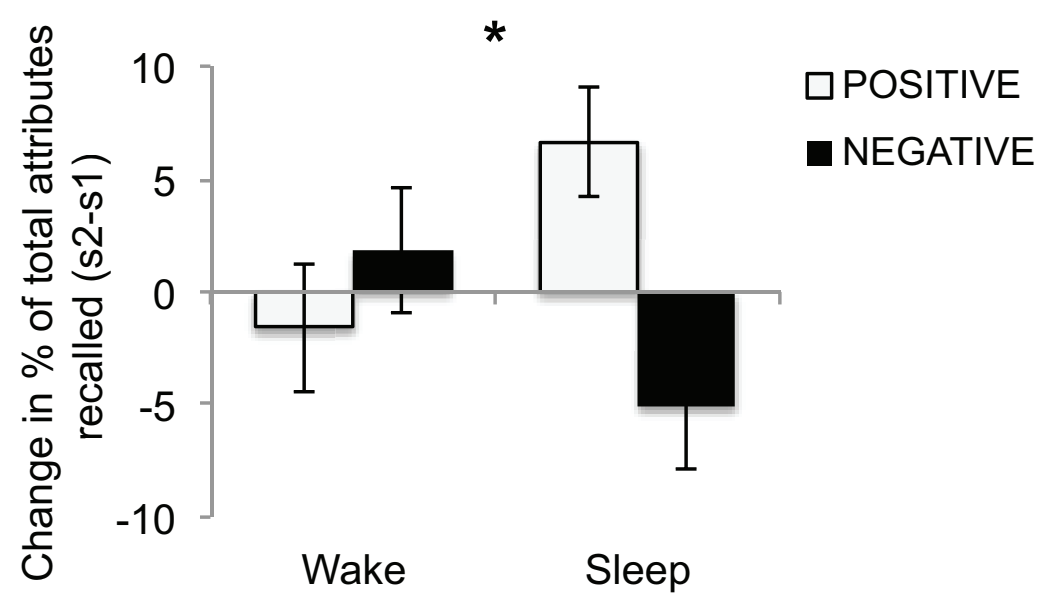

B

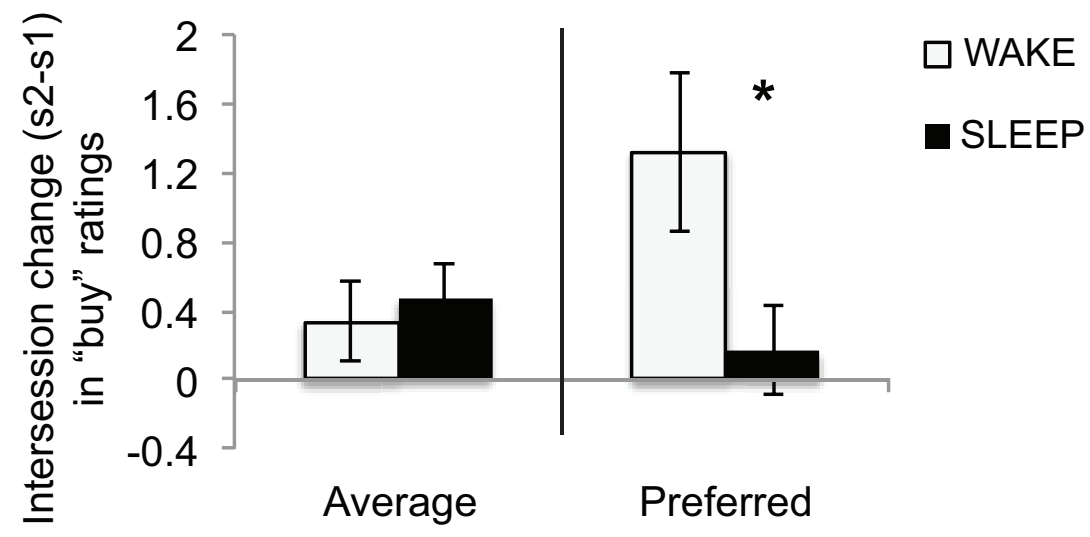

